\section{Task Shifting on Provision of Contraceptives and Abortion Services in Maternal and Reproductive Health in Sub-Saharan Africa to achieve Universal Health Coverage: A Narrative Review}

\section{Abstract}

Introduction: Improving maternal and reproductive health is a target for Sustainable Development Goals 3. Accessibility to contraceptives and abortion services is important in improving maternal and reproductive health. In 2014 in sub-Saharan Africa, over $40 \%$ of women aged $15-49$ years wanted to avoid pregnancy but only $50 \%$ or 55 million of these could access contraceptives. Shortage of skilled health workers, which is worse in Africa, has been associated with these challenges. Universal Health Coverage (UHC) is a target for world nations. To achieve UHC adequate skilled healthcare workers is one key factor needed. In 2013, globally, this shortage was 7.2 million and projected to be 12.9 million by 2035 . Task shifting has been thought as a possible solution to this shortage. Therefore, the objective of this review is to establish the outcome of task shifting in the provision of contraceptives and abortion services in sub-Saharan Africa.

Methods: A systematic approach was adopted. Medline, PsycINFO, EBM Reviews databases were searched. Google scholar and hand searching bibliographies of retrieved studies were also done. A quality appraisal using Mixed Method Appraisal Tool was done. Data were extracted using a customized form and themes identified. A narrative synthesis of the results was reported.

Results: A total of 319 records were retrieved and a set inclusion and exclusion criteria eleven studies were included in the final analysis. The quality of studies was poor as only one random control trial was retrieved limiting the quality of the evidence. Results suggest that task shifting may be a safe and effective method in improving contraceptives and abortion service accessibility in sub-Saharan Africa.

Conclusion: To cover healthcare staff shortages task shifting of contraceptive and abortion services can be considered in healthcare practices and included national human resource policies. Further research is required to cover gaps in long-term outcome these services.

Keywords: Maternal health; Reproductive health; Contraceptive services; Abortion services; Task shifting; Universal health coverage; Sub-Saharan Africa
Mate $\mathrm{M}^{1 *}$ and Thiong'o $\mathrm{F}^{2}$

1 Department of Health Services, Meru University of Science and Technology, Kenya

2 Maternal and Reproductive Health, Meru University of Science and Technology, Kenya

*Corresponding author: Mate M

fmate@must.ac.ke

Department of Health Services, Meru University of Science and Technology, Kenya

Tel: +254799529958

Citation: Mate M, Thiong'o F (2018) Task Shifting on Provision of Contraceptives and Abortion Services in Maternal and Reproductive Health in Sub-Saharan Africa to achieve Universal Health Coverage: A Narrative Review. J Contracept Stud Vol.3 No.3:20

Received: September 06, 2018; Accepted: September 10, 2018; Published: September 17,2018

\section{Introduction}

Maternal and reproductive health (MRH) are a global health concern, reasons why United Nations' Sustainable Development Goals (SDG) 3.1 aims to reduce global maternal mortality rate to less than 70 per 100,000 live births by 2030 [1]. This target is meant to improve on the global maternal mortality rate of 216 per 100,000 live births reported in 2015 [2]. The SDG 3.7 aims to ensure universal accessibility of services in sexual and reproductive healthcare, inclusive for family planning, information and education, and national strategies and programs integrating reproductive health in them [1]. Contraceptives and 
family planning are essential parts of reproductive health and by preventing pregnancy they have positive outcomes on maternal health [3]. Regardless of this knowledge, the target of increasing the use of contraceptives remains evasive [4].

Contraceptive prevalence rate, normally reported for women 15-49 years, is the proportion of women who are using (or their sexual companion is using) at least any one form of contraceptive [5]. The global contraceptive prevalence rate target is not set but aims for high percentages which in 2015 were sixty-four percent [6]. Contraceptive unmet need refers to women who are fertile and in-union or married but are not on any contraceptive method, and report desire to space births or have reached desired family size [7]. The global contraceptives unmet need target is ten percent and below, yet it is twelve percent [8]. It is important for women inclusive of adolescents to access contraceptives, safe abortion and quality post abortion services [9].

About ninety-nine percent of all maternal deaths occur in developing countries [9]. More than fifty percent of these maternal deaths occur in sub-Saharan Africa [9]. In Africa contraceptive unmet need is twenty-two percent against a global target of ten percent or below [8]. According to United Nations Population Fund, in 2014 more than forty percent of women aged 15-49 years in sub-Saharan Africa wanted to avoid pregnancy and over fifty percent of these, (55 million), were not on contraceptives and accounted for ninety-three percent of unplanned pregnancies [10]. Limited access, poor quality of available services and inadequate skilled providers contribute to these challenges [11].

The Universal Health Coverage (UHC) is a target of world nations [12]. The SDG 3.8 targets to achieve UHC including protection from financial risks and accessibility to quality essential health care services [1]. The World Health Organizations identifies adequate capacity of skilled and motivated healthcare workers as one of the factors that must be in place to achieve UHC [13]. Human resources are the foundation of any healthcare system, yet frequently they form its weakest link [14]. Shortages in health workers are exacerbated in many nations by internal distribution of highly skilled personnel that is uneven with concentration in urban areas [14]. Skilled health workers (nurses, midwives, and doctors) deficit is global standing at 7.2 million in 2013 and projected to be 12.9 million in 2035 [15]. The shortage of skilled health workers is distinctly profound in sub-Saharan Africa, which experience thirty-three percent of the global burdens of maternal, new born and child illness and sixty-seven percent of people who are living with HIV/AIDS but with a healthcare workforce that is only $2.8 \%$ [14].

Countries affected by serious deficits, especially in developing world, should consider including options like task sharing in their national health policy plans [14]. Task shifting is entrusting healthcare cadres with less training (at lower costs) with tasks normally reserved for professionals [16]. Evidence is required on outcomes of task shifting on provision of contraceptives and abortion services to guide on practice, policy and research to achieve Sustainable Development Goals.

\section{Statement of Purpose}

Scholars have carried out literature reviews in the topic on task shifting in contraceptives and abortion services. A literature review targeting studies around the world was carried out on the feasibility, safety and effectiveness of community based delivery of the depot-medroxyprogesterone acetate (DMPA) injections [17]. The review shows that trained community health workers ( $\mathrm{CHW}$ ) when trained and supervised well can provide DMPA safely. Another review was carried out with the aim of assessing how safe and effective task shifting in offering of contraceptives services, that is injections, intrauterine devices (IUDs), implants, vasectomy and tubal ligation in low and middle income countries (LMIC) [18]. The review reports that task shifting in these contraceptives may be safe and effective way to increase their access, however, due to varied certainty of the evidence there is need for further studies.

A systematic review was carried out on studies conducted around the world to find out if abortion services offered non physician health workers can be performed in a safe and effective way [19]. The review found out that safety and efficacy outcomes between nurses, physician assistants, midwives, nurses and auxiliary nurse midwives trained in medical abortion services did not differ much. However, the review reported limited evidence due to scarce random control trials and biases in cohort studies reviewed. In another review was carried out on studies worldwide with the aim of identifying how safe, efficacious or acceptable task sharing in tubal sterilization services to midlevel providers [20]. The review reports that the nine studies reviewed were of poor quality with small sample sizes to generalize the findings.

There was no literature review found that has been done on task shifting in provision of contraceptives and abortion services in sub-Saharan Africa.

\section{Objectives}

The objectives of this literature review are:

1. To establish the safety and effectiveness of task shifting services in delivery of contraceptives in sub-Saharan Africa.

2. To establish the safety and effectiveness of task shifting in offering post abortion services in sub-Saharan Africa.

\section{Methods}

This study review applied a systematic review design method with a narrative synthesis. Literature was searched from five online databases, that is, Medline, PsycINFO, EBM Reviews, PubMed Central and Google Scholar in the first week of January 2018. Other ancillary search methods were used by hand searching studies from bibliographies of studies retrieved in the online searches.

\section{Search protocol}

Population Interventions Comparators and Outcomes (PICO) were used to guide on developing search terms [21]. The target studies were on task shifting in provision of contraceptives and abortion services in MRH. Qualitative, quantitative and mixed 
methods studies were considered as appropriate. Using Ovid and PubMed a systematic search of literature was carried out in Medline, PsycINFO, and EBM Reviews databases using medical subject heading (mesh) terms and text word search. Mesh terms used were 'maternal health,' 'maternal welfare,' 'maternal health services,' 'reproductive health,' 'family planning services,' and 'contraceptives.' Text words used were 'maternal health, 'reproductive health, 'contraceptives,' 'abortion,' 'task shifting,' 'task sharing,' 'skills mix' and 'work delegation.' Truncation symbols were used to capture possible word variations in the text word search. The search was then filtered to studies published from 2005 to 2018 considering this was five years post establishment of Millennium Development Goals and onwards. Relevant studies from Google Scholar search were selected based on their title and from 2005 to 2018 publications. Relevant studies were selected from bibliographies of the retrieved studies.

\section{Quality appraisal of studies and data extraction}

Qualitative, quantitative and mixed method studies were retrieved. Mixed Methods Appraisal Tool (MMAT) was used for appraisal [22]. MMAT is a simple to use and includes appraisal for different study designs under one tool making it appropriate for appraisal of the studies with different designs. However, apart from screening questions, MMAT does not guide on what proportion of un-fulfilled criteria is relevant enough to classify a study as weak or strong. Data was extracted using a customized Microsoft Word form.

\section{Results}

The search process retrieved 150 studies from Medline, twelve studies from PsycINFO, nine studies from EMB Reviews and 26 from Google Scholar. In addition, 22 studies were handpicked from the bibliographies of the studies retrieved. This made a total of 319 studies retrieved. From these a total of 127 studies were identified by RefWorks as duplicates. The studies were further evaluated based on set inclusion and exclusion criteria. A total of 170 studies from the remaining 192 studies were eliminated based on their titles and abstracts. Thirteen studies remained and two studies were eliminated based on evaluation of their full body text. The two studies were a study by Binanga in 2016 that was a published report on a pilot study and not primarily based on task shifting the services [23]. The second excluded study by Prata et al. [24] was studying effect of reward to community health workers as a marketing strategy on injectable contraceptive distribution. Eleven studies remained for quality appraisal. The analysis steps taken are as shown in Figure 1.

Using the MMAT for appraisal it was found that of the eleven studies appraised only one study which was a random control equivalence study was of high quality. Due to the limited number of studies retrieved it was concluded that all the studies were to be included in the final review. A custom made data extraction form was used to extract data as shown in Table 1.

\section{Synthesis}

The eleven studies were synthesized and due to their heterogeneity a narrative synthesis was done using guidance on the conduct of narrative synthesis in systematic reviews [25]. The themes that were identified included provider performance and training outcomes, and clients' satisfaction.

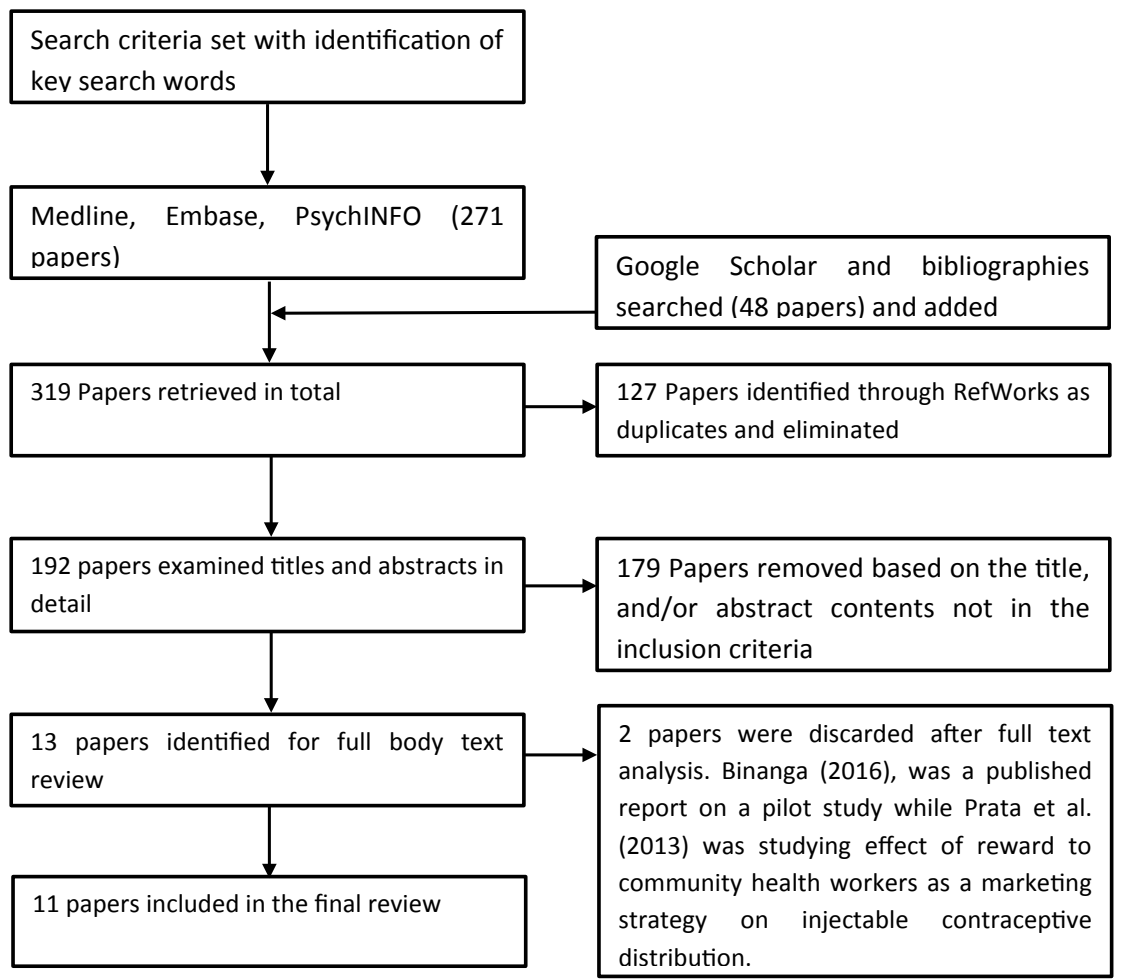

Figure 1 Literature analysis process. 
Table 1 Data extraction form.

$\begin{array}{lll}\text { Study } & \text { Country Setting } & \text { Task shifted } \\ \text { Charyeva et Nigeria } & \text { Contraceptive } \\ \text { al. [30] } & & \text { (Implants) }\end{array}$

Study setting, sampling and data collection

A sampling frame of 453 and 536 facilities was used and 166 health facilities sampled i.e. 84 in Sokoto and 82 from Bauchi. One community health extension worker (CHEW) from each of the sampled facility underwent a 2-3 weeks training on inserting implants. Feasibility was measured by surveys on the trained CHEWs, and 1 client randomly picked from each facility. Quantitative data was collected from CHEWs, clients, supply checklists and facility statistics on implants services. Qualitative data was collected from CHEWs through open-ended questio interviews. Data from these sources was analysed through triangulation.

$\begin{array}{ll}\text { Gordon Uganda } & \begin{array}{l}\text { Contraceptives } \\ \text { (Tubal ligation) }\end{array}\end{array}$

al. [32]

(Tubal ligation)

\section{A group of 4 clinical officers underwent a 6} weeks supervised training on tubal ligation. In an outreach program they conducted TL procedure on 518 women among those who wanted tubal ligation. Study outcomes measured safety of the procedure based on adverse effects, and acceptability measured based on client's satisfaction with the procedure, satisfaction with the facility and if they would recommend to a friend. Quantitative data was collected.

Guye et al. Senegal Contraceptive [31] (Implants and intrauterine devices)

Hoke et al. Madagascar Contraceptives [26] (Injections)

Two hundred and ninety health facilities in 14 regions were selected for the study. Eighty-five family planning mentors were selected from district level facilities and trained on coaching skills. The mentors were then supposed to mentor all family planning providers from the 290 lower level facilities. Five hundred and fifty-two clinical staff and 305 community health workers were trained by mentors on inserting implants. Baseline data on implants and intra-uterine devices referred to as long acting reversible contraceptive (LARC) was collected, observation checklist on provider performance and post mentorship service delivery data collected after 6-month period. In the end only 100 facilities with complete pre and post training data were included in final analysis. Quantitative data was analysed by descriptive methods.

Sixty-two community-based distribution (CBD) workers were selected from 13 administrative units. They underwent three-day training on Depot Medroxyprogesterone Acetate (DMPA) use and allowed to offer the services under supervision of nurses and doctors. Structured interviews were carried on all CBD workers. Five randomly selected clients under each $C B D$ workers were also interviewed. Statistical data was collected. Quantitative data was collected and analysed through descriptive method analysis.

Nielsen et Tanzania al. [35]

\section{Abortion services (Post abortion evacuation)}

Eleven health facilities in Geita district underwent comprehensive post-abortion care (CPAC) training on use of manual vacuum aspiration (MVA) and family planning (FP) counselling. MVA services was not previously on offer in the 11 facilities. Nine of these facilities (health centers and dispensaries) had only midlevel providers (MLP) and 2 were secondary level facilities with doctors but previously used to offer dilatation \& curettage services for CPAC. Data from May 2005 to Aug 2007 data was collected from these facilities and analysed.

\section{Findings}

After training $94.7 \%$ of the 166 CHEWs successful completed the training. They rated their skills from baseline of $6 \%$ to $93 \%$ in the end. Observation of 113 CHEWs inserting implants showed that correct performance was followed in $90 \%$ of items in checklist. Proportion of health facilities offering implants increased from $7 \%$ to 91\%. Ninety-five percent of clients were satisfied with CHEWs service on implant insertion. Only $67 \%$ of health facilities had the required supplies to insert implants at the end of the study. About $95 \%$ of CHEWs were able to receive supervisory visits ranging 1 to 8 in 6 months' period

The clinical officers were able to complete $93 \%$ of tubal ligation procedures safely. The major and moderate post procedure adverse effectively were $1.5 \%$ and $10.6 \%$ respectively. About $92-99 \%$ were satisfied with the procedure, $94-99 \%$ satisfied with the facility and $93-98 \%$ would recommend the procedure to a friend based on their experience.

After training the acceptable performance level of minimum $80 \%$ of the checklist items for correct procedure to be met on LARC insertion increased from $32 \%$ to $67 \%$ among the 552 clinical providers. The number of clients seeking LARC increased by $86 \%$ over a 6 -month period. About $64 \%$ of 290 health facilities lacked implant kits and $69 \%$ lacked intra-uterine devices kits. For purposes of the study all the 290 facilities were supplied with long acting reversible contraceptives (LARC) kits.

After the training $96 \%$ of CBD workers scored very well on the procedure questions. The four supporting NGOs sampled clients under their regions and between $91-100 \%$ of clients were satisfied with the procedures and $88-100 \%$ satisfied with CBD workers interpersonal rapport. Ninety-two percent of clients would recommend the service to a friend. Only $56 \%$ of supervisors reported having achieving monthly supervisory visit. About $36 \%$ of CBD

nd workers reported missing supplies, $28 \%$ reported long distances travelling to reach clients and $25 \%$ would require better compensation.

The total number of evacuations using MVAs rose from $0 \%$ to $66 \%$. Of these $47.9 \%$ were conducted in primary health facilities. At the end of the study $96.9 \%$ of women who underwent MVA received FP counselling and $75 \%$ took a FP method. Clients who underwent dilatation \& curettage services and received FP counselling rose from $2.5 \%$ in 2005 to $98.2 \%$ in 2007. 


\begin{tabular}{l|l|l}
\hline Study & CountrySetting & Task shifted \\
\hline $\begin{array}{l}\text { Nuccio et } \\
\text { al. [33] }\end{array}$ & Ethiopia & $\begin{array}{l}\text { Contraceptives } \\
\text { (Tubal ligation) }\end{array}$ \\
\hline Olawo et al. Kenya & $\begin{array}{l}\text { Contraceptives } \\
\text { (Injections) }\end{array}$ \\
[27] &
\end{tabular}

\section{Paul et al. Uganda}

[36]

\begin{tabular}{|l|l}
\hline & evacuation) \\
\hline $\begin{array}{l}\text { Prata et al. Ethiopia } \\
\text { 28] }\end{array}$ & $\begin{array}{l}\text { Contraceptives } \\
\text { (Injections) }\end{array}$
\end{tabular}

\section{[28]}

Stanback et Uganda

al. [29]
Study setting, sampling and data collection

The health officers were trained and supervised on tubal ligation. Three Marie Stopes InternationalEthiopia sites were purposively selected. Clients seeking tubal ligation (TL) services were offered services by the trained health officers. Total of 8 providers participated and 276 of 311 women screened were enrolled for the procedure and followed up to seven days'. Data was collected on the day of the procedure and seven days' post procedure. Descriptive analysis was done. Data on safety was analysed by proportion of adverse effects, data on feasibility analysed on proportion of checklist items adhered to and data on acceptability assessed by proportion of women that were satisfied with the procedure.

Thirty-one community health workers (CHW) from Tharaka area were selected and underwent 3 weeks training on giving DMPA injections and how to refer clients for LARC. The CHW were supervised by nurses. Data was collected on the number of clients who took over family planning methods, adverse effects reported on the infections as a measure of safety, feasibility by follow up supervision and the proportion of clients willing to have $\mathrm{CHWs}$ give them DMPA as a measure of acceptability. Data on women taking contraceptives was collected.

The study was carried out in 7 health facilities in Central Uganda where participants were purposively selected from those who offer PAC services. A total of 27 in-depth interviews were carried out on task shifting in provision of MVAs as part of PAC between midwives and doctors. Thematic analysis of the data was done.

In Ethiopia the study sampled $30 \mathrm{CBRHAs}$ and 15 health extension workers (HEW) from Tigrey region. HEW are normally qualified to give injectable contraceptives. The selected CBRHA underwent a 10-day training on the procedure and allowed to carry out the services in the community. Women independently chose who to give them the injections between CBRHAs and HEW depending on which provider they visited. The outcomes of those who visited CBRHA were compared with those of HEW. Quantitative data was collected.

Twenty community reproductive health workers (CRHW) in Nakasongola region were trained on giving injectable contraceptives. After training a study comparing the care CRHW offer and care given by nurses and midwives in health facilities was done. Overall 945 clients were recruited and 777 were followed up. After the first injection women were followed up after 13 weeks for an interview. Data collected was analysed by quantitative methods
Findings

Safety was measured by adverse events and overall had $28 \%$ had no and $72 \%$ had minor or moderate adverse effects like pain and bleeding that did not require hospital admission. On feasibility $96.9 \%$ standard clinical procedure was followed in the 176 procedures sampled. On acceptability, $97.5 \%$ of sampled clients were satisfied with the health officers $T L$ services, $98.2 \%$ of clients would recommend the procedure to a friend. However, $1.8 \%$ of clients who cited failed procedure and pain experience would not recommend the service to a friend.

Of the 1210 women who took various forms of contraceptives $69 \%$ of these methods were either started or continued using DMPA through CHW. Seventy-four percent of those who had previously used DMPA from clinics opted to receive them from $\mathrm{CHW}$. Thirty-four referrals were made in which $15 \%$ were for implants, $44 \%$ for IUD and $27 \%$ for sterilization. Use of DMPA in the area increased from $6 \%$ to $34 \%$. Of the 2,453 injections given by $\mathrm{CHW}$, No infection at injection site or needle prick was reported.

Doctors and midwives accepted that there was task shifting between the two cadres of staff in PAC. Midwives were satisfied in offering MVA services instead of turning back mothers in need or mother's conditions worsening if the doctor is not available. Doctors were positive that midwives were able to give these services in their absence.

After training CBRHA, there was no significant difference between the DMPA injection services they gave and that given by the usual HEWs. For example, $79 \%$ clients under CBRHA versus $62 \%$ under HEWs were able to go for the 3rd injection. The side effects between groups were comparable (2.1\% for CBRHA versus $0.5 \%$ for HEW) which by 3rd injection were not reported in any group. Clients were satisfied with services of CBRHA just like HEW. Fifty-two percent of clients under HEW desired to be taken over by CBRHA for home injections.

The proportion of clients seeking second injection services under CRWH was $88 \%$ and under nurses was $85 \%$. There was no significance difference in the odds of CRHW clients compared to clinic-based clients (odds ratio 1.2; 95\% confidence interval (0.8-1.9). The clients who discontinued the service due to dissatisfaction was reported in CRWHs group at $22 \%$ and in clinics groups at $40 \%$ and due to forgetting was $2 \%$ and $20 \%$ respectively. There was no significance difference in client satisfaction between those given by CRWHs versus in clinics with less than $20 \%$ of DMPA associated side effects reported in each group. 


\section{Study}

Country Setting Task shifted

Warriner et South Africa

al. [34] (Induction)
Study setting, sampling and data collection Abortion services A comparison of rates of abortion complications

\section{done by doctors versus midlevel providers (MLP)} for MVA was done in South Africa and Vietnam. Results in South Africa site showed 1160 women participated where there were 6 doctors and 6 MLPs offering the service. Women presenting in Marie Stopes International clinics for induction of abortion in first trimester were randomly assigned to a MLP or a doctor for MVA. Complications were recorded during the MVA procedure, before being discharged and during follow-up 10-14 days later. Client satisfaction data interviews were collected. Quantitative data was analysed. Data collected from clients on satisfaction was not reported in the paper.

\section{Findings}

The findings from the site in South Africa revealed overall complications were minor complications not necessitating hospital admission. In the 576 women under MLPs, 1.2\% had retained products and $0.2 \%$ compared to $0 \%$ for the 577 women under the doctors group. Rates of complications fell within the pre- determined $4.5 \%$ clinical equivalence margin. Clients reported equal satisfaction for the services offered by MLPs or by the doctors.

CBD: Community-based Distribution; CBRHA: Community-based Reproductive Health agents; CHW: Community Health Workers; CHEW:

Community Health Extension Workers; CPAC: Comprehensive Post Abortion Care; CRHW: Community-based Reproductive Health Workers;

DMPA: Depot Medroxyprogesterone Acetate; FP: Family Planning; HEW: Health Extension Workers; IUD: Intra uterine device; LARC: Long Acting Reversible Contraceptives; MLP: Midlevel Providers; MVA: Manual Vacuum Aspiration; PAC: Post Abortion Care

\section{Provision of contraceptives}

Provider performance and outcome: In Madagascar, a study found that ninety-six percent of sixty-two trained community based distributor (CBD) workers demonstrated competence on giving DMPA injections [26]. In Kenya thirty-one community health workers (CHW) were trained in Tharaka on giving contraceptive injections and this boosted DMPA use from baseline of six percent to a high of thirty-four percent [27]. A study in Ethiopia found that services of thirty trained community based reproductive health agents (CBRHA) on injections had no significant difference from the services given by fifteen health extension workers (HEW), and about seventy-nine percent of clients under CBRHA went for repeat injection compared to sixty-two percent under HEW [28]. Through a study in Uganda twenty community reproductive health workers (CRHW) were trained on injections [29]. This study found that there was no significance difference in odds of clients offered DMPA services by CRHW compared to those given by nurses (odds ratio 1.2; 95\% confidence interval 0.8-1.9). The study further reports that the proportion of clients seeking second injection was eighty-eight percent under CRHW compared to eighty-five percent under the nurses.

In Nigeria 166 community health extension workers (CHEW) were trained on implants insertion and this improved their skills from six to ninety-three percent and the proportion of health facilities offering implants increased from seven to ninety-one percent [30]. A study by in Senegal where 552 clinical providers were trained on implant insertion found their performance increased from thirty-two percent to sixty-seven percent and number of clients seeking implants increased by eighty-six percent in six month period [31]. In Uganda a study found that four clinical officers trained on tubal ligation were able to successfully complete ninety-three percent of tubal ligation on 518 women and they could handle post-procedure adverse effects [32]. Eight health officers were trained on tubal ligation in Ethiopia and conducted a safe and feasible procedure [33]. On safety the study reports that only three percent of 276 women had major adverse effects and on feasibility ninety-seven percent standard protocol was followed.
Client's satisfaction: In Madagascar it was revealed in one study that a range of between eighty-eight to one hundred percent of clients were satisfied with CBD workers giving them contraceptive injections [26]. The study further shows that ninety-two percent of the clients would recommend the service to a friend. In Ethiopia it was found that clients preferred CBRHA injections and that fifty-two percent of them opted to change from HEW to CBRHA home-based services [28]. In Uganda it was found out that dissatisfaction rates in CRHW services compared to those of HEW was twenty-two percent to forty percent respectively while client loss due to forgetting between the groups was two percent to twenty percent respectively [29]. In regard to implant insertion it was found out in Nigeria that ninety-five percent of clients receiving implants were satisfied with the service by community health extension workers ( $\mathrm{CHEW}$ ) and indicated that they would recommend the services to a friend [30]. The study on tubal ligation in Uganda reports that clients' satisfaction rates of tubal ligation procedures by clinical officers was between ninety-four and ninety-nine percent and amongst these clients ninety-three to ninety eight percent would recommend the service to a friend [32]. High client satisfaction rates were also reported in tubal ligation done by health officers in Ethiopia where ninety-eight percent of 276 clients were satisfied with the procedure [33].

\section{Provision of abortion services}

Provider's performance and outcome: In a study involving 1,160 women in South Africa it was found that only $1.4 \%$ minor complications developed from manual vacuum aspiration(MVA) done by six midlevel providers (MLP) compared to the zero percent complication in the procedures done by six doctors [34]. However, these complications were within the acceptable $4.5 \%$ clinical equivalence margin. A study in Tanzania found out that training nine MLP and two doctors led to increase in use of MVA [35]. The study reports that in primary facilities with no doctors the use of MVA increased from zero to sixty-six percent and overall forty-eight percent of all MVA were conducted in these primary facilities. An in-depth interview study in Uganda reported that midwives and doctors were task-shifting on provision of MVA services and each group was satisfied with this arrangement [36]. 
The midwives reported that the task shifting enabled clients to get emergency MVA services from them in instances where the doctors were not available hence, saving women from possible complications.

Client's satisfaction: A study in South Africa comparing equivalence of surgical abortion services offered by midlevel providers and doctors found out that clients offered the manual vacuum aspiration services by midlevel providers or doctors got equal satisfaction with the services [34].

\section{Discussion}

The review findings point that task shifting on contraceptives is a feasible, safe and acceptable way to increase accessibility in subSaharan Africa. This adds to the knowledge from earlier review on task shifting in delivery of injectable, implants, intrauterine devices, tubal ligation and vasectomy in LMIC around the world which concluded that task shifting may be an effective and safe way in advancing accessibility to contraceptives [18]. This review found that lay community workers can deliver injectable contraceptive and this adding to the knowledge from the previous review on provision of contraceptive injectable which concluded that community health workers can deliver safe contraceptive injections [17]. In regard to abortion services this review findings support that midlevel providers can offer surgical abortion and post abortion services in a safely and acceptable manner. This agrees to the review findings that sought to find evidence on which cadre can safely provide abortion services and concluded that if midlevel providers are trained they may be able to provide effective and safe early surgical abortion services [19]. It also adds to the information revealed in a review that sought to find out on task shifting in maternal and reproductive health among LMIC and part of its findings were that surgical abortion is one of the services that can safely be task shifted to midlevel providers [37].

This review confirms that task shifting may be a safe, feasible and acceptable way to increase accessibility of contraceptives and abortion services in sub-Saharan Africa, however, this evidence is limited based on the few number of articles reviewed and their quality in study methods used. The study designs used in most of the reviewed studies were not the best in practice for answering their research questions leaving a knowledge gap on the quality of evidence available. Therefore, better study designs including control trials are recommended. These findings are consistent with the review that sought to find out on the safety, efficacy and acceptability of task shifting in tubal ligation to midlevel providers [20]. The review had concluded that poor quality of the studies retrieved and sample size limited generalizability of the results and that well-designed control trials were needed for the review question to be answered well. This review also found challenges within the studies reviewed beyond the lack of skilled professionals which could influence on safety and effectiveness of provision of contraceptive and abortion services. These challenges had not been addressed in these studies. In one of the studies it was reported that there was lack of training the staff who tasks were shifted to. ${ }^{35}$ In two other studies it was reported that there was lack of support supervision for those offering the services which had had been task shifted [26,30]. In other studies in this review it was reported that lack of commodity supplies to offer the services and lack of logistics support to reach the communities requiring the services [26,30,31]. To enable increase in uptake of contraceptives there was need for contraceptives demand creation to the users which had not been addressed $[30,31]$. In one of the studies included in the review it was also noted that offering of these services faced resistance from some of the healthcare service providers mostly due to their beliefs or attitudes towards their usage [36]. Evidence on other factors limiting accessibility to contraceptive and abortion services beyond lack of skilled staff need to be studied to address and set policies on this challenge in a multipronged approach. The long term effects of tasks shifted are also not accessed. For example, proportions of failures in tubal ligations, the unintended consequences or any social-cultural issues arising from community health workers giving contraceptives injections at homes like objections from spouses. All these issues leave a knowledge gap for further studies. Therefore, there should be a more studies and multipronged approach on addressing these challenges to achieve universal health coverage.

\section{Limitations}

This review had limitations in that the search was not exhaustive in that it involved five databases. This systematic review was carried out by two authors and this limited variation or additional inputs during appraisal, data extraction and reporting as is common with systematic reviews. Due to this limitation the disagreements between the two authors during the review process were resolved through a consensus instead of using extra reviewers. The review aimed at primary studies and those retrieved were of poor quality with only one randomized control trial found. This makes the quality of evidence limited. The heterogeneity of studies limited pooling of results to carry out a meta-analysis in reporting. Therefore, the authors came up with their themes and used narrative synthesis in reporting the findings which may be biased.

\section{Conclusion}

In conclusion, to make forward steps in meeting the target of Universal Health Coverage this review makes recommendations based on healthcare practice, policy and further research.

\section{Recommendations on healthcare practice}

To achieve UHC there is need to increase accessibility of health services through task-shifting due to skilled staff shortages. With proper training and supervision delivery of contraceptives and abortion services can be offered in a safe and effective way by other cadres of healthcare providers who in normal circumstances are not qualified to offer these services. There is need for healthcare workers to change their attitudes when delivering healthcare services and limit being influenced by their own religious and cultural beliefs which influence their judgement on clients seeking to access contraceptives or abortion services.

\section{Recommendation on policy}

Countries in sub-Saharan Africa that are most affected by severe 
shortages in skilled healthcare providers should include task shifting in their national policies to allow for task shifting of services in offering these healthcare services. The governments should also offer adequate resources for training and supervision for effectiveness and safety of these services. The policy makers should also include in the policies mechanisms that involve creating awareness on contraceptive and abortion services being offered. This will create demand and hence increase utilization. There is need to include in policy mechanisms that will enable continuous support in commodity supplies to the enable uninterrupted delivery of these services. There is also need to come up with laws and policies which do not discriminate towards utilization of contraceptives and abortion services, for example, those laws that illegalize abortion.

\section{References}

1 WHO SDG 3: Ensure healthy lives and promote wellbeing for all at all ages.

2 Alkema L, Chou D, Hogan D, Zhang S, Moller BA, et al. (2016) Global, regional, and national levels and trends in maternal mortality between 1990 and 2015, with scenario-based projections to 2030: A systematic analysis by the UN maternal mortality estimation interagency group. The Lancet 387: 462-474.

3 Adongo PB, Tabong PT, Azongo TB, Phillips FJ, Sheff CM, et al. (2014) A comparative qualitative study of misconceptions associated with contraceptive use in Southern and Northern Ghana. Front Public Health 2: 137.

4 Blackstone SR, Nwaozuru U, Iwelunmor J (2017) Factors Influencing Contraceptive Use in Sub-Saharan Africa: A Systematic Review. Int Q Community Health Educ 37: 79-91.

5 WHO (2018) Contraceptive prevalence.

6 United Nations (2015) The Millennium Development Goals report 2015. New York.

7 WHO (2018) Unmet need for family planning.

8 United Nations (2017) World family planning 2017 highlights.

9 WHO (2018) Maternal mortality: Key facts.

10 UNFPA (2014) Investing in sexual and reproductive health in subSaharan Africa. Guttmacher Institute, New York.

11 WHO (2018) Family planning/contraception.

12 WHO (2018) Universal health coverage (UHC) and World Health day.

13 WHO Questions and answers on universal health coverage.

14 WHO (2010) Background paper for the global strategy for women's and children's health: Access for all to skilled, motivated, and supported health workers.

15 WHO (2013) Global health workforce shortage to reach 12.9 million in coming decades.

16 Fulton BD, Scheffler RM, Sparkes SP, Auh EY, Vujicic M, et al. (2011) Health workforce skill mix and task shifting in low income countries: A review of recent evidence. Hum Resour Health 9: 1.

17 Malarcher S, Meirik O, Lebetkin E, Shah I, Spieler J, et al. (2011) Provision of DMPA by community health workers: What the evidence shows. Contraception 83: 495-503.

\section{Recommendations on further research}

It is also notable that the number of studies retrieved was eleven which was low considering it was involving countries in subSaharan Africa. This calls for more research to be conducted in this area of research to enable wider availability of knowledge and from different countries. The evidence in this review is limited due to the majority of studies which were of poor quality. Therefore, further research is needed using quality research methods including control trials for quality results. The safety of the task shifted is reported in the short term. Therefore, long term effects and safety of these tasks shifted should also be studied in future to advice on safe practices.

18 Polus S, Lewin S, Glenton C, Lerberg PM, Rehfuess E, et al. (2015) Optimizing the delivery of contraceptives in low-and middle-income countries through task shifting: A systematic review of effectiveness and safety. Reprod Health. 12: 27

19 Renner R, Brahmi D, Kapp N (2013) Who can provide effective and safe termination of pregnancy care? A systematic review. BJOG: Int J Obstet Gynaecol 120: 23-31.

20 Rodriguez MI, Gordon-Maclean C (2014) The safety, efficacy and acceptability of task sharing tubal sterilization to midlevel providers: A systematic review. Contraception 89: 504-511.

21 Higgins JP, Green S (2011) Cochrane handbook for systematic reviews of interventions. Wiley Blackwell, John Wiley \& Sons, Ltd, Publications, US.

22 Pluye P, Robert E, Cargo M, Bartlett G, O'Cathain A, et al. Mixed methods appraisal tool (MMAT) version 2011 Proposal: A mixed methods appraisal tool for systematic mixed studies reviews. McGill University, Department of Family Medicine, Montreal, Canada.

23 Binanga A, Bertrand JT (2016) Pilot research as advocacy: The case of Sayana press in Kinshasa, Democratic Republic of the Congo. Glob Health Sci Pract 4: 542-551.

24 Prata N, Weidert K, Fraser A, Gessessew A (2013) Meeting rural demand: A case for combining community-based distribution and social marketing of injectable contraceptives in Tigray, Ethiopia. PLoS One 8 .

25 Popay J, Roberts H, Sowden A, Petticrew M, Arai L, et al. (2006) Guidance on the conduct of narrative synthesis in systematic reviews. A product from the ESRC Methods programme. Lancaster University 1: 92

26 Hoke TH, Wheeler SB, Lynd K, Green MS, Razafindravony BH, et al. (2011) Community-based provision of injectable contraceptives in Madagascar: 'task shifting' to expand access to injectable contraceptives. Health Policy Plan 27: 52-59.

27 Olawo AA, Bashir I, Solomon M, Stanback J, Ndugga BM, et al. (2013) "A cup of tea with our CBD agent: Community provision of injectable contraceptives in Kenya is safe and feasible. Glob Health Sci Pract 1 : 308-315.

28 Prata N, Gessessew A, Cartwright A, Fraser A (2011) Provision of injectable contraceptives in Ethiopia through community-based reproductive health agents. Bull World Health Organ 89: 556-564.

29 Stanback J, Mbonye AK, Bekiita M (2007) Contraceptive injections by community health workers in Uganda: A nonrandomized community trial. Bull World Health Organ 85: 768-773. 
30 Charyeva Z, Oguntunde O, Orobaton N, Otolorin E, Inuwa F, et al. (2015) Task shifting provision of contraceptive implants to community health extension workers: Results of operations research in Northern Nigeria. Global Health Sci Pract 3: 382-394.

31 Gueye B, Wesson J, Koumtingue D, Stratton S, Viadro C, et al. (2016) Mentoring, task sharing, and community outreach through the TutoratPlus approach: Increasing use of long-acting reversible contraceptives in Senegal. Global Health Sci Pract 4: 33-43.

32 Gordon-Maclean C, Nantayi LK, Quinn H, Ngo TD (2014) Safety and acceptability of tubal ligation procedures performed by trained clinical officers in rural Uganda. Int J Obstet Gynaecol 124: 34-37.

33 Nuccio O, Sendek B, Park MH, Mesele T, Okello FO, et al. (2017) Optimizing tubal ligation service delivery: A prospective cohort study to measure the task-sharing experience of Marie Stopes International Ethiopia. Health Policy Plan 32: 163-169.

34 Warriner IK, Meirik O, Hoffman M, Morroni C, Harries J, et al. (2006)
Rates of complication in first-trimester manual vacuum aspiration abortion done by doctors and mid-level providers in South Africa and Vietnam: A randomised controlled equivalence trial. Lancet 368: 1965-1972.

35 Nielsen KK, Rasch V, Lusiola G, Kanama J, Bantambya J, et al. (2009) Expanding comprehensive post abortion care to primary health facilities in Geita District, Tanzania. Afr J Reprod Health 13: 129-138.

36 Paul M, Gemzell-Danielsson K, Kiggundu C, Namugenyi R, KlingbergAllvin M (2014) Barriers and facilitators in the provision of postabortion care at district level in Central Uganda - a qualitative study focusing on task sharing between physicians and midwives. BMC Health Services Research 14: 28.

37 Dawson AJ, Buchan J, Duffield C, Homer CS, Wijewardena K (2013) Task shifting and sharing in maternal and reproductive health in lowincome countries: A narrative synthesis of current evidence. Health Policy Plan 29: 396-408. 